\title{
Exhaustivity through the Maxim of Relation
}

\author{
Matthijs Westera \\ m.westera@uva.nl \\ Institute for Logic, Language and Computation, University of Amsterdam
}

\begin{abstract}
I show that the exhaustive interpretation of answers can be explained as a conversational implicature through the Maxim of Relation, dealing with the problematic epistemic step (Sauerland, 2004). I assume a fairly standard Maxim of Relation, that captures the same intuition as Roberts' (1996) contextual entailment. I show that if a richer notion of meaning is adopted, in particular that of attentive semantics (Roelofsen, 2011), this Maxim of Relation automatically becomes strong enough to enable exhaustivity implicatures. The results suggest that pragmatic reasoning is sensitive not only to the information an utterance provides, but also to the possibilities it draws attention to. Foremost, it shows that exhaustivity implicatures can be genuine conversational implicatures. ${ }^{1}$
\end{abstract}

\section{Introduction}

Responding to a question with one of its possible answers may convey that the answer is exhaustive, i.e., that the other possible answers are false.

(1) Of blue, red and green, which colours does John like?

John likes blue. $\sim$ He doesn't like red

The exhaustive interpretation of answers is often considered a prime example of Gricean conversational implicature, but so far no theory exists that wholly explains it as such. A conversational implicature of an utterance is information that must be supposed in order to maintain the assumption that the speaker is cooperative (Grice, 1975, cf. Levinson, 2000). The typical 'Gricean' approach to the exhaustive interpretation of the response in (1) goes as follows: ${ }^{2}$

1. The speaker didn't say John likes red.

2. She should have said so, had she been able to.

${ }^{1}$ I am grateful to Jeroen Groenendijk, Floris Roelofsen, Donka Farkas, Kai von Fintel, Chris Cummins, the audiences of SPE6 (St. Petersburg), S-Circle (UC Santa Cruz), ESSLLI Student Session 2013 (Düsseldorf), LIRA (Amsterdam), TbiLLC 2013 (Gudauri) and many anonymous reviewers for very helpful comments. Financial support from the Netherlands Organisation for Scientific Research is gratefully acknowledged.

${ }^{2}$ For brevity, I will typically use the word 'know' (and, likewise, 'knowledge') as if saying 'taking oneself to know', i.e., without requiring the usual factivity associated with knowledge as being 'true belief'. 
3. She must lack the knowledge that John likes red.

...

4. She knows that John doesn't like red.

The exhaustivity in 4. is obtained from the Quantity implicature in 3. through a strengthening known as the epistemic step (Sauerland, 2004). Chierchia, Fox, and Spector (2008) argue that the epistemic step does not follow from the assumption of cooperativity, i.e., that exhaustivity is not a case of conversational implicature (instead, they defend a 'grammatical' approach to exhaustivity, which I briefly discuss in section 4). I argue, instead, that the epistemic step does follow from the assumption of cooperativity, i.e., that exhaustivity is a genuine case of Gricean conversational implicature.

My starting point is the idea that a pragmatic theory can be only as good as the stuff one feeds into it, i.e., meanings. Perhaps our pragmatic theories are fine as they are; rather, it is the underlying, classical semantics that is too coarse for an account of exhaustivity implicatures. Classical semantics models only the informative content of utterances, but the following example suggests that this is insufficient foothold for a theory of exhaustivity:

(2) Which colours does John like?

John likes \{at least blue / blue or red and blue\}. \& John doesn't like red.

The response in (1) is just as informative as the responses in (2), but only the former is interpreted exhaustively. ${ }^{3}$ Intuitively, the difference between (2) and (1) lies not in the informative content, but in the possibilities that the responses draw attention to, in particular, whether the response draws attention to the possibility that John also likes red. The responses in (2), but not (1), draw attention to this possibility, and perhaps pragmatic reasoning is sensitive to this.

The idea that pragmatic reasoning is sensitive to attentive content has been entertained before by (Ciardelli, Groenendijk, \& Roelofsen, 2009), in their account of 'might'. It entails that, if we study pragmatic phenomena, we should be using a semantics that models not only the informative content of utterances, but also their attentive content. I show that if we thus enrich the underlying semantics, the Maxim of Relation as it occurs in the literature (e.g., Roberts, 1996) automatically inherits this sensitivity. That is, the response in (1) will come out as being not entirely related to the question, because it leaves the possibility that John also likes red, unattended. This increased sensitivity of the Maxim of Relation will result in a Relation implicature that will enable us to take the epistemic step.

\footnotetext{
${ }^{3}$ Groenendijk and Stokhof (1984) already argued that exhaustivity cannot be derived through the Maxim of Quantity alone. After all, the Quantity implicature says 'this is as informative as I can safely be', hence it can never be used to strengthen what is said, as is required for the epistemic step. The contrast in (2) supports this view from a different angle: it's not only quantity of information that matters.
} 
Section 2 introduces the main building blocks - an attentive semantics and a set of fairly standard conversational maxims. Section 3 shows how exhaustivity implicatures are accounted for. Section 4 discusses the results in a broader context.

\section{Ingredients}

\subsection{Attentive semantics}

As the enriched semantic backbone for pragmatics, I adopt Roelofsen's (2011) attentive semantics, designed to model informative and attentive content. Attentive semantics is closely related to basic inquisitive semantics (Groenendijk \& Roelofsen, 2009), which is aimed at modeling informative content and inquisitive content, and unrestricted inquisitive semantics (Ciardelli, 2009; Ciardelli et al., 2009), which has been taken to model all three components at once.

In attentive semantics, the meaning of a sentence, called a proposition, is a set of sets of worlds, i.e., a set of classical propositions. The proposition $[\varphi]$ expressed by a sentence $\varphi$ is conceived of as the set of possibilities that the sentence draws attention to. The union of these possibilities corresponds to the sentence's informative content, i.e., the information provided by the sentence. Hence, attentive semantics models two semantic components - attention and information - in a single semantic object, which is called a proposition.

Let $\mathbf{W}$ be the set of all possible worlds, assigning truth values to the atomic formulae of a language of choice. Following Roelofsen (2011), I define:

- A possibility $\alpha$ is a set of worlds, $\alpha \in \wp \mathbf{W}$.

- A proposition $A$ is a set of non-empty possibilities, $A \in \wp \wp \mathbf{W}$.

$-[\varphi]$ denotes the proposition expressed by $\varphi$.

- Informative content: $|\varphi|:=\bigcup[\varphi]$

- A restricted to a set of worlds $s: A_{s}:=\{\alpha \cap s \mid \alpha \in A, \alpha \cap s \neq \varnothing\}$

The relevant fragment of propositional logic (without implication) is given in Backus-Naur Form:

Definition 1 (Syntax).

For $p$ a propositional letter, $\varphi$ formulae: $\varphi:: \perp|p| \neg \varphi|(\varphi \wedge \varphi)|(\varphi \vee \varphi)$

For all formulae $\varphi$, the proposition it expresses, $[\varphi]$, is defined recursively as:

Definition 2 (Attentive semantics).

For $p$ a proposition letter, $\varphi, \psi$ formulae:

1. $[p]=\{\{w \in \boldsymbol{W} \mid w(p)=$ true $\}\}$

2. $[\neg \varphi]=\{\overline{\bigcup[\varphi]} \mid \overline{\cup[\varphi]} \neq \varnothing\}$

3. $[\varphi \vee \psi]=([\varphi] \cup[\psi])_{|\varphi| \cup|\psi|}(=[\varphi] \cup[\psi])$

4. $[\varphi \wedge \psi]=([\varphi] \cup[\psi])_{|\varphi| \cap|\psi|}$ 
I will briefly translate this definition into natural language: atomic formulae draw attention only to one possibility, namely the possibility that the formula is true; the negation of a formula draws attention only to the single possibility where its argument is false; a disjunction draws attention to what both disjuncts draw attention to, and provides the information that at least one of them is true; and a conjunction draws attention to each possibility that a conjunct draws attention to, when restricted to the information of the other conjunct.

With this richer-than-usual semantics, entailment becomes sparser than usual:

(3) For all propositions $A, B, A$ entails $B, A \vDash B$, iff:
a. $\cup A \subseteq \cup B$; and
b. $\forall \beta \in B$, if $\beta \cap \cup A \neq \varnothing, \beta \cap \cup A \in A$

Item a. requires, just like classical entailment, that $A$ is at least as informative as $B$. Item b. requires that $A$ is, in addition, at least as attentive as $B$. That means that every possibility that $B$ draws attention to, must be a possibility that $A$ draws attention to, insofar as this is compatible with the information provided by $A$. Intuitively, $A$ entails $B$ if you can get from $B$ to $A$ by removing worlds and adding possibilities.

Despite the richer semantics, attentive semantics treats informative content fully classically, in the following sense:

Fact 1 (Classical treatment of informative content)

For all formulae $\varphi,|\varphi|(=\bigcup[\varphi])$ is its classical meaning.

This shows that, entirely in the spirit of Grice (1975), I try to account for implicatures without giving up the classical treatment of information.

\subsection{Translating the examples into logic}

To avoid having to assume anything about the semantics of 'which'-questions, I will replace the 'which'-question in (1) by the existential sentence in (4), for which the same exhaustivity implicature obtains:

(4) Among blue, red and green, there are colours that John likes.

Yes, he likes blue. $\sim$ He doesn't like red

Of course, the fact that this existential sentence behaves completely like the 'which'-question does suggest that 'which'-questions, likewise, have existential force, i.e., draw attention to the set of their non-exhaustive, mention-some answers. I will briefly return to this in section 4 .

Without loss of generality, we can furthermore assume that there exist only two colours, blue and red. Since existential quantification over a finite domain can be translated using a disjunction, this enables the following minimal translations into propositional logic:

(5) a. There are colours that John likes.

$p \vee q \vee(p \wedge q)$

b. John likes blue. 


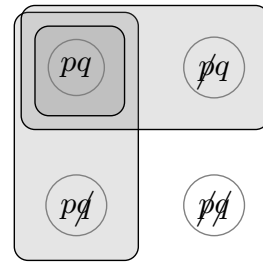

(a) $p \vee q \vee(p \wedge q)$

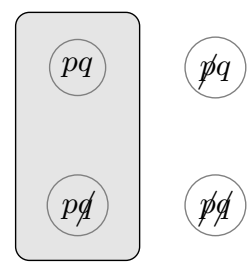

(b) $p$

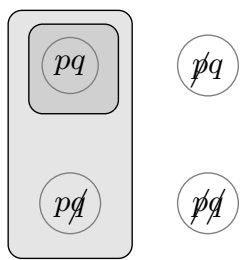

(c) $p \vee(p \wedge q)$

Fig. 1. Three propositions. Circles represent worlds, shaded areas possibilities.

c. John likes at least blue / blue or red and blue.

$p \vee(p \wedge q)$

Translations (5b) and (5c) are straightforward, where the latter is in line with Coppock and Brochhagen's (2013) account of 'at least' in inquisitive semantics.

Attentive semantics assigns to the formulae in (5) the propositions depicted in Figure 1. In the figure, circles represent possible worlds, where, e.g., $p \not$ marks the world where $p$ is true but $q$ is false, i.e., where John only likes blue. Shaded regions represent possibilities, i.e., sets of worlds. It should be clear that, in these simple examples, the possibilities that a sentence draws attention to correspond exactly to the sentence's disjuncts. This is true more generally for formulae in disjunctive normal form.

With a richer semantics, entailment is more sparse than usual. Whereas classically (5b) and (5c) would both entail (5a), now only (5c) does. This is easily seen: one cannot get from Figure 1a to Figure 1b by removing worlds or adding possibilities, due to the $|p \wedge q|$ possibility (which would have to be removed). This reflects the fact that $(5 \mathrm{~b})$, though more informative than $(5 \mathrm{a})$, is strictly less attentive. This is not the case for $(5 \mathrm{c})$.

\subsection{The maxims}

Following Grice (1975), what it means to be cooperative in a typical context is spelled out in terms of a set of conversational maxims that require the speaker to be truthful (Quality), as informative as possible and required (Quantity), relevant (Relation), and clear (Manner). Following a long tradition (at least since Groenendijk \& Stokhof, 1984; see also Roberts, 1996), the maxims of Quantity and Relation are defined in terms of a question under discussion $Q$ (which in the examples under consideration is always given as the initiative).

Definition 3 (The relevant maxims). For all propositions $A, Q$, a speaker with information state $s$ (a set of worlds), responding $A$ to $Q$, is cooperative iff:

- Quality: $s \subseteq \cup A$;

- Quantity: for all $Q^{\prime} \subseteq Q$, if $\cup A \nsubseteq \cup Q^{\prime}$ then $s \nsubseteq \cup Q^{\prime}$; 
- Relation: $A_{s} \vDash Q$; and

- Manner: the speaker thinks she is being clear, concise, etc.

I will briefly explain the maxims of Quantity and Relation.

The Maxim of Quantity The Maxim of Quantity requires that the speaker gives enough information to establish all (sets of) possibilities that she knows (or takes herself to know) contain the actual world. If a speaker is fully informed, this means that the Maxim of Quantity requires that she gives a positively exhaustive answer (i.e., an answer that decides for every possibility of the question that is the case, that it is the case), and not necessarily a positively and negatively exhaustive answer (i.e., an answer that decides for every possibility whether it is the case). This choice (i.e., that the Maxim of Quantity asks only for a positively exhaustive answer) is based on a remark by Groenendijk and Stokhof (1984) that, if it would ask for a positively and negatively exhaustive answer, then giving a non-exhaustive answer could never implicate exhaustivity, for it would implicate, instead, the speaker's inability to give an exhaustive answer. ${ }^{4}$ In addition, this choice is supported by the observation that negative answers are generally marked as deviant, requiring contrastive topic (CT) intonation, a rising pitch accent, which has been assumed to mark a change in question under discussion (Büring, 2003):

(6) A: Which colours does John like?

B: $[\text { Not }]_{C T}$ blue...

The present Maxim of Quantity explains why this is deviant: the fact that John does not like blue does not support any possibility, or set of possibilities, of the question under discussion.

The Maxim of Relation The Maxim of Relation requires that an utterance, together with some piece of information known by the speaker, entails the question under discussion. This maxim is meant to account for examples like the following:

(7) A: Did John come to the party?

B: It was raining.

Here, the response is only relevant if it is known that (and how) John's attendance depends on the rain, for instance that if it was raining, he wasn't at the party. The Maxim of Relation requires that such a dependency is known, because

${ }^{4}$ The difference between positively and negatively exhaustive answers fades if the question under discussion is a partition. Given Groenendijk and Stokhof's (1984) argument, I take this as strong evidence that, in general, questions (at least those in response to which a non-exhaustive answer would implicate exhaustivity) are not partitions; i.e., they have existential force, i.e., draw attention to their mention-some answers, rather than universal force. 
only together with such a dependency does the response entail the question (as I will formally show later).

My Maxim of Relation is slightly different from two very similar notions in the literature:

- Groenendijk and Stokhof's (1984) notion of 'pragmatic answer', which requires that the utterance, relative to the hearer's knowledge state, entails an answer to the question; and

- Roberts's (1996) 'contextual entailment', which requires that the utterance, relative to the common ground, entails an answer to the question.

First, note that my Maxim of Relation follows logically from Roberts's (1996) notion, for if the required dependency is common knowledge, then it is also the speaker's knowledge. Second, my maxim can be said to 'typically' follow from Groenendijk and Stokhof's (1984), in the following sense: if the speaker has to know that the hearer knows a dependency between answer and question, then under normal conditions of thrust, the speaker will share this knowledge. In other words, my maxim is not that different.

What my Maxim of Relation lacks, however, is a requirement that the hearer should be able to figure out how the speaker intends her response to be related to the question. I will leave such a requirement implicit (though one might think of it as part of the Maxim of Manner, which is left informal anyway), with the additional remarks that (i) compared to Groenendijk and Stokhof's (1984) and Roberts's (1996) notions, I think it is cleaner to separate this kind of 'transparency' requirement from the Maxim of Relation, and (ii) the requirements imposed by Groenendijk and Stokhof (1984); Roberts (1996) are definitely too strong: as I will show, exhaustivity implicatures are a prime example of a case where the listener need not already know how the Maxim of Relation is complied with, but, rather, can figure it out on the spot by combining it with the Quality and Quantity implicatures.

\section{$3 \quad$ Results}

\subsection{Examples}

Example (7): the rainy party Before generalizing, let us look at the examples discussed so far. Example (7), which I used solely to illustrate how the Maxim of Relation works, is accounted for as follows. Let the question (whether John was at the party) translate as $p \vee \neg p$, and the response (that it was raining) as $r$. Attentive semantics assigns to these formulae the following meanings:

$$
\begin{aligned}
& {[p \vee \neg p]=\{|p|, \overline{|p|}\}} \\
& {[r]=\{|r|\}}
\end{aligned}
$$

The response does not entail the question. For the response to be related to the question, therefore, the speaker must have some knowledge, relative to which it does entail the question. This requirement can be met in two ways: 
- The speaker thinks that if it rained, John was there $(s \subseteq \overline{|r|} \cup|p|$; the response restricted to this information yields $\{|r| \cap|p|\}$, which entails $\{|p|, \mid \overline{|p|}\}$ ); or

- The speaker thinks that if it rained, John wasn't there $(s \subseteq \overline{|r|} \cup \overline{|p|}$; the response restricted to this information yields $\{|r| \cap \mid \overline{|p|}\}$, which entails $\{|p|, \overline{|p|}\})$.

Furthermore, given that the speaker thinks that it was raining $(s \subseteq|r|)$, it follows that she must know whether John was at the party $(s \subseteq|p|$ or $s \subseteq \overline{|p|}){ }^{5}$

Example (5c): no exhaustivity Example (5c) is predicted to yield the following implicatures:

(9) 1. The speaker knows that $p(s \subseteq|p|)$

(Quality)

2. She lacks the knowledge that $q(s \nsubseteq|q|)$

(Quantity)

Because the response entails the question, it automatically complies with the Maxim of Relation as it is, i.e., no Relation implicature occurs, and as a consequence the epistemic step cannot be taken, and no exhaustivity is implicated.

Example (5b): exhaustivity! Example (5b) yields the same Quality and Quantity implicatures as (5c) (to which it is, after all, informatively equivalent). Unlike in (5c), however, here the response doesn't entail the question, hence it does not automatically comply with the Maxim of Relation. For this to be the case, the response must entail the question relative to the speaker's information state. This can be complied with in two ways:

- The speaker thinks that if John likes blue, he likes red $(s \subseteq \overline{|p|} \cup|q|$; the response restricted to this information yields $\{|p| \cap|q|\}$, which entails the question); or

- The speaker thinks that if John likes blue, he doesn't like red $(s \subseteq \overline{|p|} \cup \overline{|q|}$; the response restricted to this information yields $\{|p| \cap \overline{|q|}\}$, which entails the question).

Combining this disjunctive implicature with the Quality and Quantity implicatures yields the following:

1. The speaker knows that $p(s \subseteq|p|)$

(Quality)

2. She lacks the knowledge that $q(s \nsubseteq|q|)$

(Quantity)

\footnotetext{
${ }^{5}$ This shows that, in this case, the Relation implicature in fact clashes with the Quantity implicature: for if the speaker really knows whether John was at the party, then why didn't she just say so? As a consequence, the example will likely trigger additional implicatures to explain this clash, e.g., that the speaker wants to test the hearer's knowledge. (Understandably, therefore, the example under discussion is more natural with a final rising pitch contour, which has been argued to convey uncertain relevance/relatedness (Ward \& Hirschberg, 1985).) Regardless of this, the example suffices for merely illustrating how the Maxim of Relation works.
} 
That is, exhaustivity is derived as a conversational implicature.

At a more intuitive level, the following is going on in this example. For any possibility of the question that the speaker, in her response, leaves unattended (in this case $|q|$ ), the Maxim of Relation, with its increased sensitivity due to attentive semantics, requires that the speaker knows whether it or its negation follows from the information that she did give (i.e., the information that $|p|)$ ). Or in other words, the Maxim of Relation requires that the speaker is opinionated about every possibility she leaves unattended. This implicated opinionatedness is what enables the epistemic step. ${ }^{6}$

\subsection{General results}

While the implicatures due to the maxims of Quantity and Quality are quite easy to spell out, the Maxim of Relation is rather more complex - and yet that is where the key to exhaustivity lies. In order to better understand it, the implicatures due to the Maxim of Relation can be generally characterized as follows:

\section{(10) Relation implicature}

A cooperative speaker with information state $s$, responding $A$ to $Q$, complies with the Maxim of Relation iff:

a. $s \subseteq \overline{\cup A} \cup \cup Q$; and

b. for all $\gamma \in Q: s \subseteq \overline{\cup A} \cup \bar{\gamma}$, or there is an $\alpha \in A$ s.t. $s \subseteq(\overline{\gamma \cap \cup A} \cap \bar{\alpha}) \cup(\gamma \cap \cup A \cap \alpha)$

Item (a) requires that the speaker knows the material implication from $A$ to $Q$, which ensures that the response together with the speaker's information, is at least as informative as the question. Item (b) requires that the response, together with the speaker's information, is at least as attentive as the question. This amounts to the following: for each possibility that the question draws attention to, the speaker knows either that it is incompatible with the response, or that, when restricted to the response and the speaker's information, it coincides with a possibility $\alpha$ that the response draws attention to.

For responses that select exactly one of the possibilities of the question, which are interesting from the viewpoint of exhaustivity, this amounts to the following more readable result:

\footnotetext{
6 The speaker can be opinionated in two ways, and in the case of exhaustivity, the Quality and Quantity implicatures together settle how (something which did not happen in the rainy party example). This shows that, as mentioned above, it would be too strict to require that the hearer already knows how a response is related to the question; in the case of exhaustivity, the Quality and Quantity implicatures enable her to figure it out.
} 


\section{Relation implicature for singleton answer}

A cooperative speaker with information state $s$, responding $\{\alpha\}$ to $Q$ for some $\alpha \in Q$, complies with the Maxim of Relation iff:

for all $\gamma \in Q, s \subseteq \bar{\alpha} \cup \bar{\gamma}$; or $s \subseteq \bar{\alpha} \cup \gamma$.

That is, for every possibility that the question draws attention to, the speaker should know how it depends on the information provided - which is exactly the kind of Relation implicature that enables the epistemic step. ${ }^{7}$

\section{Discussion}

Using standard conversational maxims, but assuming that they are sensitive to attentive content, I have shown that exhaustivity implicatures are genuine conversational implicatures. The current section discusses further links to the literature.

\subsection{Against the opinionatedness assumption}

In the literature, the epistemic step is often taken by invoking the contextual assumption that the speaker is opinionated (e.g., Sauerland (2004); Schulz and Rooij (2006); Spector (2007), but basically every 'Gricean' approach since Mill (1867)).

1. She lacks the knowledge that $q$

2. She knows either $q$ or $\neg q$

(Quantity)

(Opinionatedness)

3. She knows $\neg q$

Aside from being very unsatisfying (it demotes exhaustivity to a case of underspecification), these approaches predict that removing the opinionatedness assumption from the context would cancel the exhaustivity implicature, which is not in fact borne out:

(12) A: I'm probably asking the wrong person - you're probably not opinionated about this at all - but of red, green, blue, and yellow, which colours does John like?

B: He likes red and blue. $\leadsto$ He doesn't like green, yellow.

This shows that the opinionatedness is not a contextual assumption, but, rather, something conveyed by the speaker herself. Indeed, in my approach, the Quality and Relation implicatures together entail that the speaker is opinionated about every possibility she leaves unattended. To my awareness, my approach is the first Gricean approach of exhaustivity that can account for this.

\footnotetext{
${ }^{7}$ In the future I hope to give such general descriptions of the Relation implicature also for cases where the response is any (potentially non-singleton) subset of the question, as these are all and only the cases that may yield exhaustivity implicatures. However, so far the results for such question-response pairs have not turned out any more readable than the general characterisation in (10), and I will omit them.
} 


\subsection{Other suitable semantics}

Attentive semantics is not the only suitable backbone for a pragmatic account of exhaustivity, and thinking of these richer meanings in terms of attentive content is not the only way. For instance, in Westera (2012) used Ciardelli's (2009) unrestricted inquisitive semantics (I chose attentive semantics this time because it is conceptually better motivated). Minimally, however, a suitable semantic backbone for a pragmatic theory of exhaustivity must lack the absorption laws:

Definition 4 (Absorption laws). $\varphi \wedge(\varphi \vee \psi) \equiv \varphi \equiv \varphi \vee(\varphi \wedge \psi)$

Is is seen very easily: (5b) and (5c) can be semantically distinguished only if the absorption laws do not hold, and this is precisely the distinction to which the maxims should be sensitive for an account of exhaustivity.

\subsection{Alternatives and scales}

All existing Gricean approaches in the literature treat exhaustivity as an answer to the question: 'why did the speaker not utter a more informative alternative?'. The problem is that mere ignorance is sufficient excuse for not giving a more informative answer, hence no exhaustivity implicature is predicted. One way to think of my approach, however, is that exhaustivity is treated as an answer to the question: 'why did the speaker not utter a more attentive alternative?'. Because one can draw attention to possibilities without committing to them (such as $|p \wedge q|$ in (5)c), mere ignorance is insufficient reason for not uttering the more attentive alternative, and therefore something stronger is implicated: exhaustivity.

One can (but need not) think of the present approach as relying on scales of alternatives, that are ordered by entailment just as in the original work of Horn (1984), with the difference that entailment is now sensitive to attentive content. However, the notion of a scale is unnecessary: what counts as an alternative in a particular context is fully determined by what counts as cooperative in that context. This is defined by the maxims, which do not themselves refer to this kind of alternative. It also entails that speakers need not reason in terms of alternatives; they can just apply the maxims directly (as I did for the examples above). This perspective might be relevant for experimental work on the processing of implicatures.

\subsection{Against the grammatical approach to exhaustivity}

In the introduction I mentioned the 'grammatical approach' to exhaustivity. This approach aims to attribute exhaustivity to the presence of optional, silent 'exhaustivity operators' in the grammar. The motivation for this kind of approach, which is certainly dispreferred compared to a Gricean approach for reasons of parsimony, comes mainly from two claims (as formulated by Chierchia et al. (2008)). First, that the Gricean approach cannot solve the epistemic step. Second, that there are cases of exhaustivity that seem to 'arise from' an embedded 
position (such as the 'not all' interpretation of an existential embedded under a universal quantifier) that a Gricean theory cannot account for. I will not spend too much time on this debate, but I will briefly show why I think that both claims, in fact, bite their owner (see also Geurts, 2011).

Regarding the first claim, I have of course shown at length that a Gricean approach can, in fact, account for the epistemic step. As for the grammatical approach, it is completely unclear to me how it could avoid the epistemic step, as its followers seem to believe it could. For how can a speaker convey, or a listener know, when and where an optional, silent exhaustivity operator should be inserted? The answer, I think, can only be: through some kind of Gricean inference schema - but this makes the grammatical approach just as susceptible to the problem of the epistemic step as existing Gricean approaches.

The second claim is of course susceptible to the same criticism: how does a speaker convey, or a hearer infer, that a silent, optional exhaustivity operator should be inserted in an embedded position? Again, the only answer can be: through some kind of Gricean inference schema. (Ironically, this shows, quite unintended by those who have been gathering evidence of embedded implicatures, that a Gricean theory must be able to account for 'embedded implicatures'.) It has never been shown that a Gricean theory cannot in principle account for implicatures that target embedded positions. The general intuition seems to be that because Gricean reasoning is post-compositional (it operates on speech acts), it cannot have access to sub-sentential structure, which would be required for 'embedded implicatures'. But clearly this doesn't hold. For instance, in attentive semantics, the possibilities in a proposition reflect the disjuncts in a sentence, and the maxims have access to those, even if the disjuncts are embedded under, say, a conjunction. More generally, why would a post-compositional pragmatic reasoner ever wilfully ignore the constituents of a sentence, or its derivational history, or the words that were used?

A wide range of cases of 'embedded implicature' is reported on in the literature (and understandably so: an approach as un-parsimonious as the grammatical one is hungry for evidence seemingly in their favour), and space does not permit a more detailed discussion. I am confident that my theory already accounts for some cases of embedded implicature, but also that it cannot yet account for all cases. At the same time, I am confident that the grammatical approach does not account for any of them.

\section{References}

Büring, D. (2003). On d-trees, beans, and accents. Linguistics and Philosophy, 26, 511-545.

Chierchia, G., Fox, D., \& Spector, B. (2008). The grammatical view of scalar implicatures and the relationship between semantics and pragmatics. In C. Maienborn, P. Portner, \& K. von Heusinger (Eds.), Semantics: An international handbook of natural language meaning (Vol. 2, p. 22972332). Mouton de Gruyter. 
Ciardelli, I. (2009). Inquisitive semantics and intermediate logics. (Master Thesis, University of Amsterdam)

Ciardelli, I., Groenendijk, J., \& Roelofsen, F. (2009). Attention! Might in inquisitive semantics. In S. Ito \& E. Cormany (Eds.), Proceedings of Semantics and Linguistic Theory (SALT XIX).

Coppock, E., \& Brochhagen, T. (2013). Raising and resolving issues with scalar modifiers. (Submitted to Semantics and Pragmatics)

Geurts, B. (2011). Quantity implicatures. Cambridge University Press.

Grice, H. (1975). Logic and conversation. In P. Cole \& J. Morgan (Eds.), Syntax and semantics (Vol. 3, pp. 41-58).

Groenendijk, J., \& Roelofsen, F. (2009). Inquisitive semantics and pragmatics. In J. M. Larrazabal \& L. Zubeldia (Eds.), Meaning, content, and argument: Proceedings of the ILCLI international workshop on semantics, pragmatics, and rhetoric.

Groenendijk, J., \& Stokhof, M. (1984). Studies on the semantics of questions and the pragmatics of answers. Unpublished doctoral dissertation, University of Amsterdam.

Horn, L. (1984). Towards a new taxonomy of pragmatic inference: Q-based and R-based implicatures. In D. Schiffrin (Ed.), Meaning, form, and use in context (pp. 11-42). Georgetown University Press.

Levinson, S. (2000). Presumptive meanings. MIT press.

Mill, J. S. (1867). An examination of sir william hamilton's philosophy.

Roberts, C. (1996). Information structure in discourse. In J. Yoon \& A. Kathol (Eds.), Osu working papers in linguistics (Vol. 49, pp. 91-136). Ohio State University.

Roelofsen, F. (2011). Information and attention. (Manuscript, ILLC University of Amsterdam)

Sauerland, U. (2004). Scalar implicatures in complex sentences. Linguistics and Philosophy, 27(3), 367-391.

Schulz, K., \& Rooij, R. van. (2006). Pragmatic meaning and non-monotonic reasoning: The case of exhaustive interpretation. Linguistics and Philosophy, 29, 205-250.

Spector, B. (2007). Scalar implicatures: Exhaustivity and gricean reasoning. In M. Aloni, A. Butler, \& P. Dekker (Eds.), Questions in dynamic semantics (pp. 225-250). Elsevier.

Ward, G., \& Hirschberg, J. (1985). Implicating uncertainty: the pragmatics of fall-rise intonation. Language, 61.4, 747-776.

Westera, M. (2012). Meanings as proposals: a new semantic foundation for Gricean pragmatics. (Presented at SemDial 2012) 